\title{
Characterization of Metal Cluster Catalysts in Mesoporous Silicas
}

\author{
N. S. Hondow*, G. A. Koutsantonis* and M. Saunders** \\ * Chemistry, School of Biomedical and Chemical Sciences, The University of Western Australia, \\ Australia. \\ ** Centre for Microscopy and Microanalysis, The University of Western Australia, Australia.
}

Western Australia (WA) has vast natural gas reserves, worth more than AUD\$1.5 billion per year in export earnings. The first two phases of the development of the North West Shelf Gas Project in Western Australia have consisted of the commercial sale of household gas in WA and the liquefaction and export of gas. However, there is potential for the Fischer-Tropsch process to be implemented to obtain more useful products from the natural gas, such as transportation fuels. The type of catalyst used in this process can determine the range and amount of products collected. At present there are no uniquely selective catalysts available that make this process an economic viability.

Catalysts are in development where the active metals are attached to an inorganic support. The support, a type of mesoporous silica, is part of a family known as the M41S materials, which were initially developed in 1992 [1]. These materials are characterized by large surface areas and pore sizes between 15 and $80 \AA$ in size.

Metal clusters of high nuclearity containing metals known to be active in the Fischer-Tropsch process are attached to the mesopores bringing active catalytic sites to the new materials. The resulting materials can be characterized by a variety of techniques, such as transmission electron microscopy (TEM), x-ray diffraction (XRD), surface area measurements, small angle x-ray scattering (SAXS), etc.

Using a JEOL 3000F field emission gun TEM (FEG-TEM), a variety of studies of the catalytic materials have been undertaken. Conventional bright field imaging has been used to characterize the initial, unmodified M41S materials, to determine the size and regularity of the pore systems. While it is difficult to obtain accurate pore size measurements from this approach, inter-pore spacings can be obtained.

Metal clusters, formed by organometallic synthesis, are reacted with the porous silicas in an attempt to disperse the metal clusters within the pores. The interest in these new materials lies in establishing whether or not the metal particles have attached to the mesoporous silica and, if they have, it is important to discover where they are attached, what size they are and to confirm what elements are present. Bright field images of the reacted materials still show the pore structure, but it is difficult to clearly identify the attached metal clusters. However, this information can be established using alternative imaging techniques such as scanning transmission electron microscopy (STEM) and high resolution transmission electron microscopy (HRTEM), and microanalysis techniques such as energy dispersive $\mathrm{x}$-ray mapping (EDS mapping). 
The ability to obtain atomic number contrast has made STEM especially useful in studying these materials. With high angle annular dark field (HAADF) imaging the metal clusters, which are of a much heavier molecular weight than the surrounding support material, appear much brighter than the lighter mesoporous silica matrix. Combining this with EDS mapping confirms that the brighter and more intense sections in the HAADF STEM images are in fact due to the metal particles. Similarly HRTEM can be used to image the structure of the metal particles on the silica support. Using these techniques the size, structure and distribution of the metal particles has been determined.

The TEM studies are proving to be invaluable in the study of these materials, with images showing well-ordered pores and the attached metal clusters produced. STEM and EDS mapping studies have been especially useful for these materials, combining elemental analysis with atomic number contrast imaging.

\section{References}

[1] Beck, J. S. et al.. J. Am. Chem. Soc. 114 (1992) 10834.

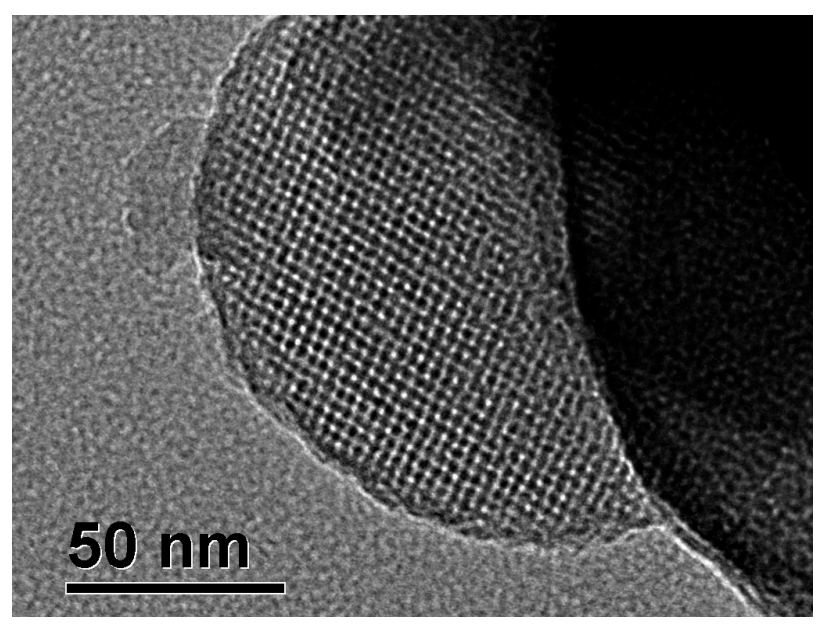

FIG. 1. Bright field image of MCM-48 mesoporous silica showing the regular pore distribution.

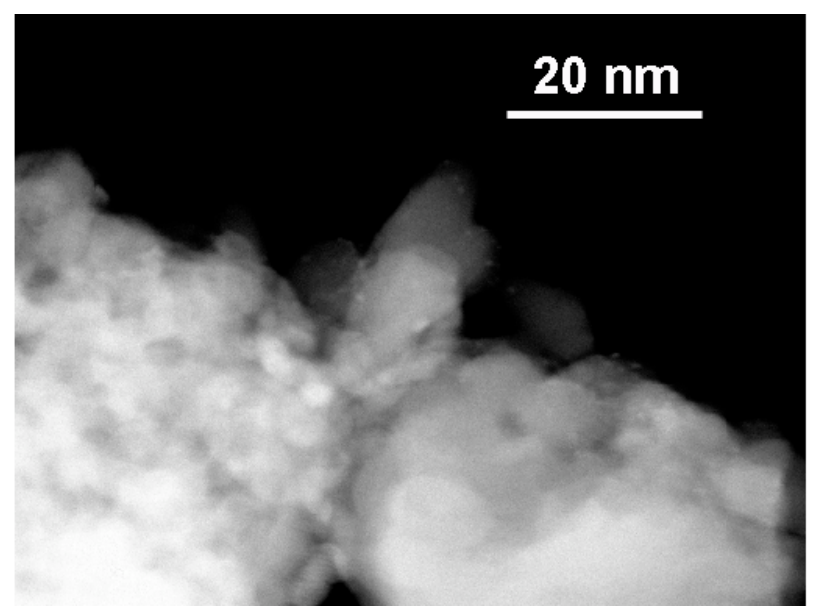

FIG. 2. HAADF STEM image of MCM-48 with attached $~ 2 \mathrm{~nm}$ ruthenium metal clusters. 